\title{
RESEARCH
}

Open Access

\section{The impact of physical activity on healthy ageing trajectories: evidence from eight cohort studies}

\author{
Darío Moreno-Agostino ${ }^{1 * \dagger} \mathbb{D}$, Christina Daskalopoulou ${ }^{1 \dagger}$, Yu-Tzu Wu' ${ }^{1}$ Artemis Koukounari ${ }^{2}$, Josep Maria Haro ${ }^{3,4}$,
} Stefanos Tyrovolas ${ }^{3,4}$, Demosthenes B. Panagiotakos ${ }^{5}$, Martin Prince ${ }^{1}$ and A. Matthew Prina ${ }^{1}$

\begin{abstract}
Background: Research has suggested the positive impact of physical activity on health and wellbeing in older age, yet few studies have investigated the associations between physical activity and heterogeneous trajectories of healthy ageing. We aimed to identify how physical activity can influence healthy ageing trajectories using a harmonised dataset of eight ageing cohorts across the world.

Methods: Based on a harmonised dataset of eight ageing cohorts in Australia, USA, Mexico, Japan, South Korea, and Europe, comprising 130,521 older adults $\left(M_{\text {age }}=62.81, S D_{\text {age }}=10.06\right)$ followed-up up to 10 years ( $M_{\text {follow-up }}=$ 5.47, $S D_{\text {follow-up }}=3.22$ ), we employed growth mixture modelling to identify latent classes of people with different trajectories of healthy ageing scores, which incorporated 41 items of health and functioning. Multinomial logistic regression modelling was used to investigate the associations between physical activity and different types of trajectories adjusting for sociodemographic characteristics and other lifestyle behaviours.

Results: Three latent classes of healthy ageing trajectories were identified: two with stable trajectories with high (71.4\%) or low (25.2\%) starting points and one with a high starting point but a fast decline over time (3.4\%). Engagement in any level of physical activity was associated with decreased odds of being in the low stable (OR: $0.18 ; 95 \% \mathrm{Cl}: 0.17,0.19)$ and fast decline trajectories groups (OR: $0.44 ; 95 \% \mathrm{Cl}: 0.39,0.50)$ compared to the high stable trajectory group. These results were replicated with alternative physical activity operationalisations, as well as in sensitivity analyses using reduced samples.

Conclusions: Our findings suggest a positive impact of physical activity on healthy ageing, attenuating declines in health and functioning. Physical activity promotion should be a key focus of healthy ageing policies to prevent disability and fast deterioration in health.
\end{abstract}

Keywords: Growth mixture modelling, Lifestyle behaviours, Health metric, Data harmonisation, Physical activity

\footnotetext{
* Correspondence: dario.moreno@kcl.ac.uk

${ }^{+}$Darío Moreno-Agostino and Christina Daskalopoulou contributed equally to this work.

'Department of Health Service and Population Research, King's College

London, Institute of Psychiatry, Psychology and Neuroscience, David

Goldberg Centre, De Crespigny Park, London SE5 8AF, UK

Full list of author information is available at the end of the article
}

C C The Author(s). 2020 Open Access This article is licensed under a Creative Commons Attribution 4.0 International License, which permits use, sharing, adaptation, distribution and reproduction in any medium or format, as long as you give appropriate credit to the original author(s) and the source, provide a link to the Creative Commons licence, and indicate if changes were made. The images or other third party material in this article are included in the article's Creative Commons licence, unless indicated otherwise in a credit line to the material. If material is not included in the article's Creative Commons licence and your intended use is not permitted by statutory regulation or exceeds the permitted use, you will need to obtain permission directly from the copyright holder. To view a copy of this licence, visit http://creativecommons.org/licenses/by/4.0/ The Creative Commons Public Domain Dedication waiver (http://creativecommons.org/publicdomain/zero/1.0/) applies to the data made available in this article, unless otherwise stated in a credit line to the data. 


\section{Background}

Ageing has been associated with an increased risk of non-communicable diseases, frailty [1] and disability [2]. To address economic, health and social care burdens related to these adverse health conditions, maintenance of good health in later life has become a key priority for ageing research and health policy planning. In this regard, the latest World Report on Ageing and Health by the World Health Organization (WHO) [3] has provided a framework for the study and promotion of healthy ageing. In this report, WHO defined healthy ageing as the "process of developing and maintaining the functional ability that will enable older people to do the things that matter to them", and this process is not homogeneous in the population [4].

Physical activity promotion has been suggested to reduce the risk of developing non-communicable diseases [5] and the healthcare expenditure [6], and to increase the satisfaction with one's life and with the ageing process [7]. Evidence from epidemiological studies has also shown that physical activity is strongly associated with healthier ageing trajectories $[8,9]$, better cognitive function trajectories [10] and improved frailty trajectories [11]. In particular, a recent meta-analysis [12] has summarised the findings from 23 longitudinal studies focusing on the associations between physical activity and healthy ageing and suggested that physically active older adults (defined by any levels of exercises or activities) had almost $40 \%$ increased odds of experiencing healthy ageing compared to their non-physically active counterparts. However, there was a high heterogeneity in the conceptualisation of healthy ageing, with different studies using different combinations of variables related to physical performance (functioning and disability), diseases, mental health, and survival to a specific age [12]. Moreover, most existing studies mainly focused on population average trajectories of healthy ageing and did not consider the existence of subgroups in the older population that may exhibit different trajectories than the majority of it [3]. This might be due to limited sample size and statistical power to detect subgroups of different trajectories within a single study population. To provide novel insights on how physical activity can influence trajectories of healthy ageing in different populations, it is necessary to incorporate data from multiple cohort studies and use comparable measures for physical activity and healthy ageing.

In this study, we aim to investigate the impact of physical activity on trajectories of healthy ageing, while taking into account sociodemographic factors and other correlated lifestyle factors, by using a harmonised dataset of eight longitudinal cohort studies across the world. Given the strength of large sample sizes, we identified various types of healthy ageing trajectories in the study population and examined their relationships with different levels of physical activity.

\section{Methods \\ Study sample}

This study was based on the Ageing Trajectories of Health: Longitudinal Opportunities and Synergies (ATHLOS) project (http://athlosproject.eu/). The aim of the ATHLOS project was to harmonise data from 17 international ageing cohort studies [13] and to investigate trajectories of healthy ageing and their potential determinants in different older populations. Data harmonisation is a technique where data from different studies and with different format are transformed and merged together in order to produce one cohesive dataset. Bringing together 17 ageing cohorts across the world, the ATHLOS harmonised dataset highlights several advantages of data harmonisation: first, it allows researchers to increase the size of the study population and hence the statistical power of the analyses; second, it enables the investigation of those trajectories and any potential determinants independently from the older adults' settings and backgrounds while adjusting the analyses for differences in the source of the data, thus providing more generalisable evidence. The ATHLOS consortium followed the Maelstrom Research guidelines [14]. Harmonisation of variables across studies was based on an iterative process of consensus of experts, which is transparently documented and publicly available harmonisation reports (https://github.com/athlosproject/athlos-project.github.io/). Further details on the included cohorts and the harmonised variables is also available in the cohort profile of the ATHLOS project [13]. To carry out longitudinal analyses and identify different trajectories of healthy ageing, we focused on cohorts with three or more available waves. This included the Australian Longitudinal Study of Ageing (ALSA), the English Longitudinal Study of Ageing (ELSA), the Study on Cardiovascular Health, Nutrition and Frailty in Older Adults in Spain (ENRICA), the Health and Retirement Study (HRS), the Japanese Study of Ageing and Retirement (JSTAR), the Korean Longitudinal Study of Ageing (KLOSA), the Mexican Health and Aging Study (MHAS) and the Survey of Health Ageing and Retirement in Europe (SHARE). In the present study, we excluded participants with information on health only at a single time point. The analytic sample comprised 130,521 individuals from 26 countries (i.e. Australia, Austria, Belgium, Czech Republic, Denmark, Estonia, France, Germany, Greece, Hungary, Ireland, Israel, Italy, Japan, Luxembourg, Mexico, Netherlands, Poland, Portugal, Slovenia, South Korea, Spain, Sweden, Switzerland, United Kingdom, and USA), whereas the excluded sample comprised 78,279 individuals. 


\section{Measures}

\section{Healthy ageing score}

Based on the WHO framework healthy ageing [3, 15], the ATHLOS healthy ageing score was constructed by using a two-parameter logistic item response theory (IRT) model with 41 items related to intrinsic capacity and functional ability (see Table S1, Supplementary Material). Heterogeneities in the cohort-specific datasets were analysed and addressed to obtain the common scale. The IRT model converged successfully with an excellent fit (RMSEA $=0.03, \mathrm{TLI}=0.99$, and CFI $=0.99$ ), and had a marginal reliability of 0.83 . The estimated latent scores obtained for each participant at each time point were rescaled to a range between 0 and 100, with higher numbers indicating better health status.

\section{Physical activity}

Two harmonised variables were used to assess physical activity at baseline: frequency of vigorous exercise and frequency of less vigorous exercise throughout the week (in days). Both variables had five categories (never, once per week, 2/3 times/days per week, 4-5 times/days per week, and 6-7 times/days per week) that were recoded from the original variables in which participants were asked for the number/frequency of "vigor sessions" (ALSA), "vigorous physical activities" (HRS), "strenuous activity" (JSTAR), "exercise” (KLOSA), or "sports or activities that are vigorous" (SHARE), regarding vigorous exercise; or "less vigor sessions" (ALSA), "light physical activities" (HRS), "light exercise" (JSTAR), or "activities requiring a moderate level of energy" (SHARE), regarding less vigorous exercise. The last two categories of the harmonised variables were grouped into 4+ times/days per week in this study. As not all included studies had this information, we created an aggregated variable of physical activity (yes/no) to minimise missingness. To create the latter variable, we considered the following harmonised questions (the corresponding response options appear between parentheses): level of physical activity (high, fair, low, not at all), frequency of vigorous and less vigorous exercise (never, once per week, 2/3 times/days per week, $4+$ times/days per week), engagement in vigorous exercise during the last 2 weeks (yes, no), frequency of vigorous exercise activities in the last 2 weeks (number), and time spent doing vigorous exercise in the last 2 weeks (in minutes). Participants were categorised as physically active if at least one of the following criteria was met: had "high" or "fair" level of physical activity; engaged in less vigorous or more vigorous exercise $2+$ times per week or more; engaged in vigorous exercise (either with a "yes" to the frequency question, or with an answer different from zero to the question on the time spent doing vigorous exercise), or had 5 or more time vigorously exercised in the last 2 weeks. The presence of these different questions by study is shown in Table S2 (Supplementary Material).

\section{Covariates}

Covariates included age, gender, study, education, wealth, smoking and alcohol consumption. Education was categorised into three groups (primary or less, secondary and tertiary education) based on qualification. Wealth was measured using income and financial information and divided into quintiles (1st: least) within each cohort. As in the case of physical activity, we harmonised measures of alcohol consumption and smoking that allowed the least amount missing data across studies. Thus, we considered if participants reported ever having smoked or consumed alcohol either at baseline or any of the follow-up waves.

\section{Statistical analyses}

To investigate the heterogeneity in the longitudinal trajectories of health, we employed the framework of growth mixture modelling (GMM) [16], which allows identifying unobserved groups (latent classes) of individuals who exhibit different patterns of health change over time. Since most cohorts had an investigation period up to 10 years and carried out follow-up waves every 2 years, here we focused on the first 10 years of follow-up and built trajectories by biennial intervals (year 0, 2, 4, 6, 8 and 10). Hence, mean elapsed time across waves was 2 years (variance is not available).

Following Ram and Grimm indications [16], we performed a single-class model (i.e. latent growth curve model) to find the best representation of change in the overall sample (linear or quadratic change). We used the best fitting model [lower Bayesian Information Criterion $(\mathrm{BIC})$ ] as baseline model against which we compared subsequent models with different number of latent classes, ranging from two to five. We computed these models considering either linear or quadratic change. To decide on the final number of latent classes (i.e. trajectories), we employed the Bayesian Information Criterion (BIC), the sample-size adjusted BIC (SABIC), entropy values, the Lo-Mendel-Rubin likelihood ratio test (LMRLRT), and the sample size of the smallest class (no less than $1 \%$ of the total sample in any class) $[17,18]$. The entropy of the model was prioritised as selection criteria in order to optimise the separation between the classes [16]. The models were estimated using maximum likelihood with errors robust to non-normality and nonindependence of observations (MLR) [19], and missing data were assumed to be missing at random (MAR). Moreover, we used 500 random sets of starting values for the parameters, along with 250 final optimisations, to prevent the models from converging on a local solution. In order to balance the flexibility of the models with the 
estimation ability, residual variances and covariances of the growth parameters were estimated but constrained to be equal across latent classes to avoid estimation problems. Alternative specifications of the within-class heterogeneity such as Latent Class Growth Analysis, in which within-class heterogeneity is constrained to be zero, were not considered due to their inability to reflect the expected individual heterogeneity in the healthy ageing process $[3,4]$.

Once the model was selected, we used a three-step approach to estimate the association of the three physical activity variables (i.e. aggregated physical activity, frequency of vigorous exercise, and frequency of less vigorous exercise) with each latent class. In this approach, 1) the latent class model is estimated a priori without accounting for the predictor variables; 2) observations are then assigned to the most likely class using the latent class posterior distribution obtained in step 1; and 3) a new model is then estimated in order to assess the impact of the predictor variables on the class membership, fixing the measurement error to the values obtained in step 2 [20, 21]. This procedure accounts for misclassification in the second step, and the error assignments based on the highest posterior probabilities are reduced. All multinomial logistic regression models were adjusted for other lifestyle behaviours (i.e. ever smoking and drinking), as well as for baseline age, gender, study (i.e. ALSA, ELSA, ENRICA, HRS, JSTAR, KLOSA, MHAS, and SHARE), within-country household wealth quintile (1st and 2nd quintiles vs the rest), and education level (i.e. primary or less than primary, secondary, and tertiary). In these models, participants with missing data in any of the covariates were excluded from the analyses.

Since some studies did not have information on the frequency of vigorous (i.e. ELSA, ENRICA, and MHAS) or less vigorous exercise (i.e. ELSA, ENRICA, KLOSA, and MHAS), we performed a set of sensitivity analyses to investigate the robustness of our findings. In these sensitivity analyses, we performed the selected GMM model in two reduced samples (sensitivity analysis A: studies with information on vigorous exercise; sensitivity analysis B: studies with information on less vigorous exercise) and checked whether the results regarding the trajectories found and their relationship with physical activity were replicated. The Guidelines for Reporting on Latent Trajectory Studies [22] were used, and the checklist is included in the Table S3 (Supplementary Material). Data management and descriptive analyses were performed using Stata SE 14.2, whereas GMM models were performed in Mplus 8.1. The code is available from the corresponding author upon request.

\section{Results}

Trajectories of healthy ageing

A total of 130,521 participants had information in at least two measurement points. The average number of observations was $3.19(S D=1.39)$, and the mean duration of the follow-up was 5.47 years $(S D=3.22)$. Table 1 summarises characteristics of the analytic and excluded samples at baseline. Although some differences across included and excluded samples achieved statistically significance (to be expected due to the large sample size), the only meaningful effect size was found in the study variable, where SHARE had a higher proportion of excluded cases due to single observations from the refresh cohorts. Regarding the analytic sample, mean scores on health by years of follow-up were $70.21(S D=16.46$, skewness $=-0.41$, kurtosis $=2.70$ ) for baseline, 69.02 $(S D=17.30$, skewness $=-0.44, \quad$ kurtosis $=2.77)$ for 2 years, $68.69(S D=17.53$, skewness $=-0.42$, kurtosis $=$ $2.81)$ for 4 years, $67.25(S D=17.83$, skewness $=-0.33$, kurtosis $=2.74)$ for 6 years, $66.99(S D=18.45$, skewness $=$ -0.31 , kurtosis $=2.50)$ for 8 years, and $65.78(S D=$ 18.43 , skewness $=-0.27$, kurtosis $=2.50$ ) for 10 years.

Table 2 provides the BIC, SABIC, adjusted LMR-LRT results, and entropy values for the one to five latent classes models. Models allowing for quadratic change showed better fit than those only allowing for linear change. The three-class solution with quadratic growth term showed the highest entropy (0.72). The trajectories resulting from all these models are depicted in Figures S1, S2, S3, S4, S5, S6, S7, S8, S9 (Supplementary Material). Systematically, the models with three or more classes showed the existence of a subgroup comprising around $3-4 \%$ of the population displaying a high starting point and a fast decline over time. Increasing the number of classes beyond three resulted on spliting the remaining classes, displaying a relatively stable trajectory, into additional subgroups with different starting points but parallel trajectories. The three-class model with quadratic growth was selected as the optimal solution due to its higher entropy.

The trajectories resulting from the selected model are depicted in Fig. 1. Latent class groups were named according to their trajectory patterns. Latent class 3 comprised the majority of the population (green line, 71.4\%), displaying a high stable level of health throughout the whole period (high stable). Participants in latent class 1 (blue line, 25.2\%) displayed a low level of health in the beginning and almost no change across the follow-ups (low stable). Participants in latent class 2 (red line, 3.4\%) showed a baseline level of health similar to the high stable class, but a severe deterioration over time (fast decline). The specific estimates for the model and each of the classes are displayed in Table 3 (upper section). 
Table 1 Baseline characteristics of analytic and excluded samples

\begin{tabular}{|c|c|c|c|c|c|}
\hline & & $\begin{array}{l}\text { Analytic sample } \\
(N=130,521)\end{array}$ & $\begin{array}{l}\text { Excluded sample } \\
(N=78,279)\end{array}$ & $t / x^{2}$ & Effect size \\
\hline Age, M (SD) & & $62.80(10.06)$ & $62.31(11.68)$ & $10.29^{* * *}$ & 0.05 \\
\hline \multirow[t]{3}{*}{ Gender, N (\%) } & Female & $72,312(55.40)$ & 40,632 (51.91) & 0.32 & 0.001 \\
\hline & Male & $58,063(44.49)$ & $32,453(41.46)$ & & \\
\hline & Missing & $146(0.11)$ & $5194(6.64)$ & - & - \\
\hline \multirow[t]{6}{*}{ Wealth, N (\%) } & 1st quintile & $26,406(20.23)$ & $13,702(17.50)$ & $266.04^{* * *}$ & 0.04 \\
\hline & 2nd quintile & $25,419(19.48)$ & $13,064(16.69)$ & & \\
\hline & 3rd quintile & $24,760(18.97)$ & $13,663(17.45)$ & & \\
\hline & 4th quintile & $24,040(18.42)$ & $14,162(18.09)$ & & \\
\hline & 5th quintile & $23,657(18.13)$ & 14,892 (19.02) & & \\
\hline & Missing & $6239(4.78)$ & $8796(11.24)$ & - & - \\
\hline \multirow[t]{4}{*}{ Education, N (\%) } & Less than primary/primary & $43,469(33.3)$ & $22,181(28.34)$ & $488.55^{* * *}$ & 0.05 \\
\hline & Secondary & $62,821(48.13)$ & $38,425(49.09)$ & & \\
\hline & Tertiary & $21,834(16.73)$ & $14,666(18.74)$ & & \\
\hline & Missing & $2397(1.84)$ & $3007(3.84)$ & - & - \\
\hline \multirow[t]{8}{*}{ Study, N (\%) } & ALSA & $1843(1.41)$ & $244(0.31)$ & $27,000^{* * *}$ & 0.36 \\
\hline & ELSA & $14,483(11.1)$ & $4006(5.12)$ & & \\
\hline & ENRICA & $2513(1.93)$ & $6(0.01)$ & & \\
\hline & HRS & $32,934(25.23)$ & $4382(5.60)$ & & \\
\hline & JSTAR & $3695(2.83)$ & $3573(4.56)$ & & \\
\hline & KLOSA & $8928(6.84)$ & $1326(1.69)$ & & \\
\hline & MHAS & $12,647(9.69)$ & $8719(11.14)$ & & \\
\hline & SHARE & $53,478(40.97)$ & $56,023(71.57)$ & & \\
\hline Health score, M (SD) & & $70.21(16.46)$ & $70.38(17.81)$ & $-2.05^{*}$ & -0.0004 \\
\hline \multirow[t]{3}{*}{ Physical activity (aggregated), N (\%) } & No & $49,622(38.02)$ & $21,999(28.10)$ & $1300^{* * *}$ & 0.08 \\
\hline & Yes & $80,001(61.29)$ & $50,555(64.58)$ & & \\
\hline & Missing & $1397(0.69)$ & $5725(7.31)$ & - & - \\
\hline \multirow[t]{5}{*}{ Frequency of vigorous physical activity, N (\%) } & Never & $42,244(32.37)$ & $26,326(33.63)$ & $3300^{* * *}$ & 0.15 \\
\hline & Once per week & $20,738(15.89)$ & $12,865(16.43)$ & & \\
\hline & 2/3 per week & $7129(5.46)$ & $882(1.13)$ & & \\
\hline & $4+$ per week & $23,789(18.23)$ & $19,278(24.63)$ & & \\
\hline & Missing & $36,621(28.06)$ & $18,928(24.18)$ & - & - \\
\hline \multirow[t]{5}{*}{ Frequency of less vigorous physical activity, N (\%) } & Never & $11,751(9.00)$ & $8371(10.69)$ & $5100^{* * *}$ & 0.19 \\
\hline & Once per week & $16,840(12.90)$ & $11,984(15.31)$ & & \\
\hline & 2/3 per week & $10,311(7.90)$ & $1145(1.46)$ & & \\
\hline & 4+ per week & $44,556(34.14)$ & $36,324(46.40)$ & & \\
\hline & Missing & $47,063(36.06)$ & 20,455 (26.13) & - & - \\
\hline \multirow[t]{3}{*}{ Ever smoker*, N (\%) } & No & $62,168(47.63)$ & $40,439(51.66)$ & $487.34^{* * *}$ & 0.05 \\
\hline & Yes & $66,817(51.19)$ & $35,508(45.36)$ & & \\
\hline & Missing & $1536(1.18)$ & $2332(2.98)$ & - & - \\
\hline \multirow[t]{3}{*}{ Ever drinker*, N (\%) } & No & 39,019 (29.89) & 29,797 (38.07) & $1800^{* * *}$ & 0.09 \\
\hline & Yes & $91,023(69.74)$ & $46,260(59.10)$ & & \\
\hline & Missing & $479(0.37)$ & $2222(2.84)$ & - & - \\
\hline
\end{tabular}

Note. All variables correspond to participants' baseline, except for ever drinker and ever smoker, which correspond to the whole period of participation. Effect size estimates for age and health score correspond to Cohen's $d$; the remaining effect size estimates correspond to Cramer's $V$, and have been computed with non-missing data only. M: mean, SD: standard deviation, $\mathrm{N}$ : number of cases, $t$ : Student's $t$ test; $x^{2}$ : chi-square test. *: $p<.05$; ***: $p<.001$ 
Table 2 Fit statistics of the Latent Growth Curve Model and Growth Mixture Models

\begin{tabular}{|c|c|c|c|c|c|}
\hline & \multicolumn{5}{|c|}{ Linear growth - Number of latent classes } \\
\hline & 1 class & 2 classes & 3 classes & 4 classes & 5 classes \\
\hline $\begin{array}{l}\text { Loglikelihood } \\
\text { (N of parameters) }\end{array}$ & $-1,661,740(6)$ & $-1,657,724(9)$ & $-1,656,300(12)$ & $-1,655,481(15)$ & $-1,654,823(18)$ \\
\hline BLRT & & $8032.7^{* * *}$ & $2846.4^{* * *}$ & $1639.3^{* * *}$ & $1314.5^{* * *}$ \\
\hline Adj. LMR-LRT & & $7811.6^{* * *}$ & $2768.1^{* * *}$ & $1594.2^{* * *}$ & $1278.3^{* * *}$ \\
\hline $\mathrm{BIC}$ & $3,323,552$ & $3,315,554$ & $3,312,743$ & $3,311,139$ & $3,309,860$ \\
\hline SABIC & $3,323,532$ & $3,315,526$ & $3,312,705$ & $3,311,091$ & $3,309,803$ \\
\hline Entropy & & 0.644 & 0.692 & 0.661 & 0.690 \\
\hline \multicolumn{6}{|l|}{ Group size (\%) } \\
\hline Class 1 & 100 & 73.5 & 4.1 & 54.1 & 17.2 \\
\hline Class 2 & & 26.5 & 25.4 & 9.9 & 2.1 \\
\hline Class 3 & & & 70.5 & 3.6 & 43.1 \\
\hline Class 4 & & & & 32.4 & 34.1 \\
\hline \multirow[t]{3}{*}{ Class 5} & & & & & 3.5 \\
\hline & \multicolumn{5}{|c|}{ Quadratic growth - Number of latent classes } \\
\hline & 1 class & 2 classes & 3 classes & 4 classes & 5 classes \\
\hline Loglikelihood ( $\mathrm{N}$ of parameters) & $-1,660,737(10)$ & $-1,656,703(14)$ & $-1,655,111(18)$ & $-1,654,139(22)$ & \\
\hline BLRT & & $8067.8^{* * *}$ & $3184.6^{* * *}$ & $1943.9^{* * *}$ & \\
\hline Adj. LMR-LRT & & $7900.1^{* * *}$ & $3118.4^{* * *}$ & $1903.5^{* * *}$ & \\
\hline $\mathrm{BIC}$ & $3,321,592$ & $3,313,571$ & $3,310,434$ & $3,308,537$ & \\
\hline SABIC & $3,321,560$ & $3,313,527$ & $3,310,377$ & $3,308,467$ & \\
\hline Entropy & & 0.644 & 0.722 & 0.668 & \\
\hline \multicolumn{6}{|l|}{ Group size (\%) } \\
\hline Class 1 & 100 & 73.5 & 25.2 & 34.2 & \\
\hline Class 2 & & 26.5 & 3.4 & 12.2 & \\
\hline Class 3 & & & 71.4 & 3.3 & \\
\hline Class 4 & & & & 50.3 & \\
\hline Class 5 & & & & & \\
\hline
\end{tabular}

Note. ${ }^{* *} p<.001$. The model with quadratic growth and 5 classes provided an improper solution and therefore is not displayed in the table. BLRT Bootstrap likelihood ratio test; Adj. LMR-LRT Adjusted Lo-Mendell-Rubin likelihood ratio test; BIC Bayesian Information Criterion; SABIC Sample-size-Adjusted Bayesian Information Criterion

\section{Impact of physical activity on the latent classes}

Table 3 (lower section) shows the odds ratios from the multinomial logistic regression models of the latent classes on physical activity adjusted for age, gender, study, wealth, education level, ever smoking, and ever drinking. Participants engaging in vigorous or less vigorous exercise throughout the week, compared with physically inactive participants, had significantly reduced likelihood of being in the low stable and fast decline latent-classes than in the high stable latentclass. Similar findings were also observed for the aggregated variable of physical activity. For instance, participants that were characterised as physically inactive had 5.56 times the odds $\left(O R_{\text {active }}=0.18,95 \% C I\right.$ $[0.17,0.19])$ of being in the low stable latent class compared to the high stable latent class; and 2.27 times the odds $\left(O R_{\text {active }}=0.44,95 \% C I[0.39,0.50]\right)$ of being in the fast decline latent class.

Table 4 and Table 5 display the results of the sensitivity analyses performed with the reduced samples [i.e. with the studies that had information on frequency of vigorous (A) and less vigorous exercise (B), respectively]. These analyses showed similar results regarding the number and the pattern of the health trajectories and their associations with physical activity.

\section{Discussion}

Main findings

Using a harmonised dataset of eight ageing cohorts across the world, we identified three types of healthy ageing trajectories (high stable, low stable and fast decline) and investigated how physical activity in older age 


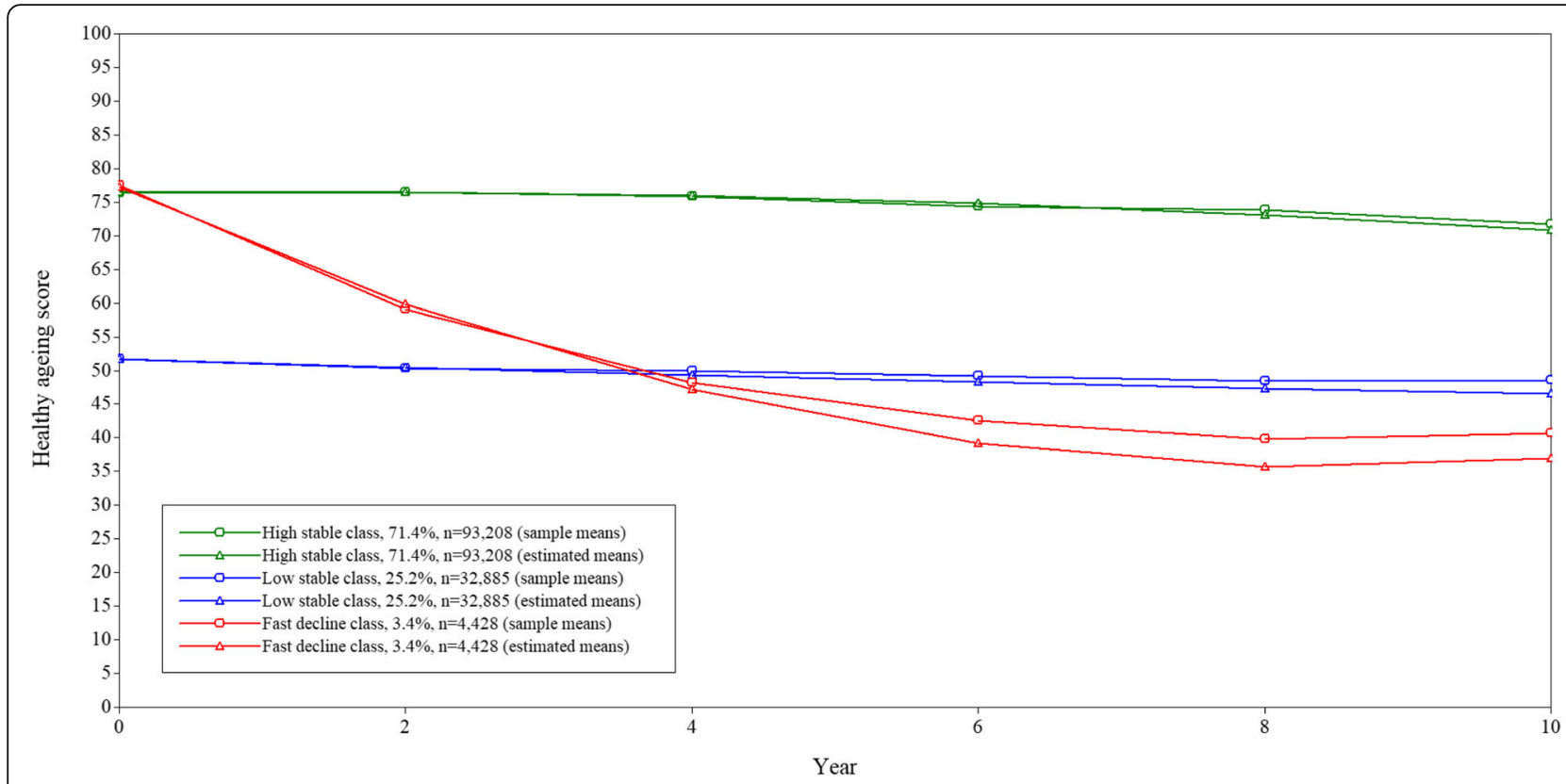

Fig. 1 Trajectories of health according to the three latent classes Growth Mixture Model

Table 3 Estimates for the three latent classes Growth Mixture Model of healthy ageing and multinomial logistic regressions coefficients

\begin{tabular}{|c|c|c|c|}
\hline \multicolumn{4}{|l|}{ Latent classes $(n=130,521)$} \\
\hline & High stable & Low stable & Fast decline \\
\hline n (\%) & $93,208(71.4)$ & $32,885(25.2)$ & $4428(3.4)$ \\
\hline Mean Intercept (SE) & $76.47(0.08)$ & $51.72(0.12)$ & $77.18(0.31)$ \\
\hline Mean Linear Slope (SE) & $0.15(0.03)$ & $-0.64(0.05)$ & $-9.81(0.57)$ \\
\hline Mean Quadratic Slope (SE) & $-0.07(0.003)$ & $0.01(0.01)$ & $0.58(0.06)$ \\
\hline Variance Intercept (SE) & $83.02(1.04)$ & & \\
\hline Variance Linear Term (SE) & $2.78(0.17)$ & & \\
\hline Variance Quadratic Term (SE) & $0.03(0.002)$ & & \\
\hline Covariance Intercept-Linear Term (SE) & $-0.58(0.30)$ & & \\
\hline Covariance Intercept-Quadratic Term (SE) & $-0.01(0.03)$ & & \\
\hline Covariance Linear-Quadratic Terms (SE) & $-0.21(0.02)$ & & \\
\hline \multicolumn{4}{|l|}{ Physically active $(n=120,712)$} \\
\hline No & Reference class & Ref. & \\
\hline Yes, OR $(95 \% \mathrm{Cl})$ & & $0.18[0.17,0.19]$ & $0.44[0.39,0.50]$ \\
\hline \multicolumn{4}{|l|}{ Vigorous physical activity $(n=90,451)$} \\
\hline Never & Reference class & Ref. & \\
\hline Once per week, OR (95\% Cl) & & $0.22[0.20,0.24]$ & $0.43[0.34,0.56]$ \\
\hline 2/3 times per week, OR (95\% Cl) & & $0.18[0.16,0.21]$ & $0.25[0.16,0.39]$ \\
\hline 4+ times per week, OR (95\% Cl) & & $0.11[0.10,0.12]$ & $0.65[0.53,0.80]$ \\
\hline \multicolumn{4}{|c|}{ Less vigorous physical activity ( $n=80,707$ ) } \\
\hline Never & Reference class & Ref. & \\
\hline Once per week, OR (95\% Cl) & & $0.17[0.15,0.19]$ & $0.26[0.20,0.34]$ \\
\hline 2/3 times per week, OR $(95 \% \mathrm{Cl})$ & & $0.07[0.05,0.12]$ & $0.11[0.07,0.16]$ \\
\hline 4+ times per week, OR (95\% Cl) & & $0.07[0.06,0.07]$ & $0.23[0.18,0.28]$ \\
\hline
\end{tabular}

Note. $\mathrm{Cl}$ Confidence interval; OR Odds ratio; SE Standard error. All multinomial logistic regression models are adjusted for age, gender, study, wealth, education, smoking, and alcohol drinking 
Table 4 Estimates for the sensitivity analysis A: three latent classes Growth Mixture Model of healthy ageing and multinomial logistic regressions coefficients with reduced sample (studies with information on vigorous activity)

\begin{tabular}{|c|c|c|c|}
\hline \multicolumn{4}{|l|}{ Latent classes $(n=100,878)$} \\
\hline & High stable & Low stable & Fast decline \\
\hline n (\%) & $73,112(72.5)$ & $24,303(24.1)$ & $3463(3.4)$ \\
\hline Mean Intercept (SE) & $77.06(0.09)$ & $51.88(0.15)$ & $78.67(0.36)$ \\
\hline Mean Linear Slope (SE) & $0.17(0.03)$ & $-0.82(0.05)$ & $-9.16(0.67)$ \\
\hline Mean Quadratic Slope (SE) & $-0.07(0.003)$ & $0.03(0.005)$ & $0.49(0.07)$ \\
\hline Variance Intercept (SE) & $83.79(1.15)$ & & \\
\hline Variance Linear Term (SE) & $2.80(0.20)$ & & \\
\hline Variance Quadratic Term (SE) & $0.03(0.002)$ & & \\
\hline Covariance Intercept-Linear Term (SE) & $-0.07(0.32)$ & & \\
\hline Covariance Intercept-Quadratic Term (SE) & $-0.06(0.03)$ & & \\
\hline Covariance Linear-Quadratic Terms (SE) & $-0.22(0.02)$ & & \\
\hline \multicolumn{4}{|l|}{ Physically active ( $n=96,701)$} \\
\hline No & Reference class & Ref. & \\
\hline Yes, OR (95\% Cl) & & $0.16[0.15,0.17]$ & $0.40[0.34,0.46]$ \\
\hline \multicolumn{4}{|l|}{ Vigorous physical activity $(n=90,451)$} \\
\hline Never & Reference class & Ref. & \\
\hline Once per week, OR $(95 \% \mathrm{Cl})$ & & $0.22[0.20,0.24]$ & $0.42[0.32,0.54]$ \\
\hline 2/3 times per week, OR (95\% Cl) & & $0.19[0.16,0.21]$ & $0.22[0.13,0.35]$ \\
\hline 4+ times per week, OR (95\% Cl) & & $0.12[0.11,0.13]$ & $0.65[0.52,0.82]$ \\
\hline \multicolumn{4}{|c|}{ Less vigorous physical activity $(n=\mathbf{8 0 , 7 0 7 )}$} \\
\hline Never & Reference class & Ref. & \\
\hline Once per week, OR (95\% Cl) & & $0.17[0.15,0.19]$ & $0.23[0.17,0.31]$ \\
\hline 2/3 times per week, OR $(95 \% \mathrm{Cl})$ & & $0.08[0.07,0.09]$ & $0.09[0.06,0.14]$ \\
\hline 4+ times per week, OR (95\% Cl) & & $0.07[0.06,0.07]$ & $0.21[0.17,0.27]$ \\
\hline
\end{tabular}

Note. Cl: confidence interval; OR: odds ratio; SE: standard error. All multinomial logistic regression models are adjusted for age, gender, study, wealth, education, smoking, and alcohol drinking

was associated with these different types of trajectories. Our study suggests a positive impact of physical activity on supporting healthy ageing. Engagement in any levels of physical activity was associated with decreased odds of being in low stable or fast decline groups of healthy ageing trajectories.

\section{Strengths and limitations}

Our study has several methodological strengths in comparison with previous research. The ATHLOS harmonised dataset covered participants from different backgrounds and the longitudinal data provided rich information on changes in health and functioning over 10 years. The large sample size and harmonised variables allowed us to explore various types of healthy ageing trajectories and their relationships with physical activity in different populations. This may increase the generalisability of the findings. Instead of focusing on presence of selected diseases or impairments, we conceptualised healthy ageing using multiple indicators of health, physical and cognitive functioning and generated a common measure across multiple cohorts.

Our findings should be considered in the light of limitations. Firstly, harmonisation as a process is retrospective and the initial studies were not designed to be harmonised. This reflects on the amount of missing data in some of the physical activity variables. To try to overcome this limitation, 1) we conducted sensitivity analyses to assess potential differences in the number and the characteristics of the latent classes due to missing values on these physical activity variables. We found that the shape and the size of the latent groups were similar across all analyses, suggesting the robustness of the results. 2) We also created the physical activity aggregated variable and employed it along with the other two physical activity variables to assess the potential impact of these different operationalisations on the associations. Even though there were some differences in the effect size 
Table 5 Estimates for the sensitivity analysis B: three latent classes Growth Mixture Model of healthy ageing and multinomial logistic regressions coefficients with reduced sample (studies with information on less vigorous activity)

\begin{tabular}{|c|c|c|c|}
\hline \multicolumn{4}{|l|}{ Latent classes $(n=91,950)$} \\
\hline & High stable & Low stable & Fast decline \\
\hline n (\%) & $61,774(67.2)$ & $25,461(27.7)$ & $4715(5.1)$ \\
\hline Mean Intercept (SE) & $78.21(0.08)$ & $52.51(0.13)$ & $78.92(0.23)$ \\
\hline Mean Linear Slope (SE) & $0.42(0.04)$ & $-0.84(0.05)$ & $-7.97(0.49)$ \\
\hline Mean Quadratic Slope (SE) & $-0.09(0.004)$ & $0.03(0.005)$ & $0.41(0.05)$ \\
\hline Variance Intercept (SE) & $75.32(1.15)$ & & \\
\hline Variance Linear Term (SE) & $2.80(0.23)$ & & \\
\hline Variance Quadratic Term (SE) & $0.03(0.002)$ & & \\
\hline Covariance Intercept-Linear Term (SE) & $-1.07(0.36)$ & & \\
\hline Covariance Intercept-Quadratic Term (SE) & $0.04(0.04)$ & & \\
\hline Covariance Linear-Quadratic Terms (SE) & $-0.23(0.02)$ & & \\
\hline \multicolumn{4}{|l|}{ Physically active $(n=87,806)$} \\
\hline No & Reference class & Ref. & \\
\hline Yes, OR (95\% Cl) & & $0.18[0.17,0.20]$ & $0.41[0.36,0.46]$ \\
\hline \multicolumn{4}{|l|}{ Vigorous physical activity ( $n=81,556$ ) } \\
\hline Never & Reference class & Ref. & \\
\hline Once per week, OR (95\% Cl) & & $0.27[0.25,0.28]$ & $0.43[0.35,0.53]$ \\
\hline 2/3 times per week, OR (95\% Cl) & & $0.21[0.19,0.24]$ & $0.20[0.14,0.30]$ \\
\hline $4+$ times per week, OR $(95 \% \mathrm{Cl})$ & & $0.14[0.13,0.15]$ & $0.58[0.47,0.71]$ \\
\hline \multicolumn{4}{|c|}{ Less vigorous physical activity ( $n=80,707$ ) } \\
\hline Never & Reference class & Ref. & \\
\hline Once per week, OR (95\% Cl) & & $0.21[0.19,0.23]$ & $0.32[0.25,0.40]$ \\
\hline 2/3 times per week, OR (95\% Cl) & & $0.10[0.09,0.11]$ & $0.15[0.11,0.20]$ \\
\hline 4+ times per week, OR (95\% Cl) & & $0.09[0.08,0.10]$ & $0.24[0.20,0.29]$ \\
\hline
\end{tabular}

Note. CI Confidence interval; OR Odds ratio; SE Standard error. All multinomial logistic regression models are adjusted for age, gender, study, wealth, education, smoking, and alcohol drinking

estimates, the conclusions did not change based on the measure used: people that engaged in some physical activity had higher chances to be included in the group with better health. In line with this first limitation, the aggregated physical activity variable was created ad hoc in the present study to allow for the inclusion of cohort studies that used different operationalisations of physical activity. However, the sensitivity analyses performed using different categorisations of physical activity achieved similar results, thus providing evidence on the robustness of the findings involving this aggregated variable. Additionally, the information on physical activity was based on self-reported items, which may have been affected by response biases. These limitations prevent us, for instance, from providing detailed insights about the specific amount of physical activity that related to the membership to the better healthy ageing trajectory. Although we controlled for behavioural factors such as smoking and alcohol drinking, we could not control for the potential confounding effect of diet quality in our analyses [23] since that information was not available in the majority of the included studies. Moreover, the sample size in smallest latent class (i.e. fast decline) was relatively low, which led to wider confidence intervals. Finally, although recent research on low- and middle-income countries has found results that are consistent with the evidence presented in our study $[9,24]$, it is important to note that most of the countries included in this study were high-income countries. As a result of these limitations, extra caution is needed when generalising these findings.

\section{Interpretation of findings}

The results from our study suggest that physically inactive older adults were more likely to exhibit worse trajectories of health with age than those that engaged in some form of physical activity. This corresponds to the literature and highlights the important 
role of being physically active in healthy ageing [12, 25]. Instead of focusing on presence of diseases or dichotomised health outcomes [25], our study shows the existence of subgroups within the older population exhibiting different trajectories of health and provides evidence on how physical activity is related to those different trajectories after adjusting for socioeconomic factors, smoking, drinking, and data source (i.e. study). It is true that variation in the healthy ageing trajectories may be the result of specific determinants (i.e. physical activity as our findings indicate) or due to the heterogeneity across countries or research settings. However, previous research in sub-studies of the ATHLOS harmonised dataset [i.e. 10/66 study [24] and MHAS study [9]] has suggested similar trajectories of healthy ageing and a similar effect of physical activity to those. Notably, the study focused on the MHAS cohort [9] adopted a similar analytical approach and identified four types of healthy ageing trajectories (high stable, moderate stable, low stable, and fast decline) in over the 10 year follow-up period and reported that physical activity was associated with lower odds of being in low stable and fast decline groups. Taking these previous research evidence and the findings of the present study altogether, it may be assumed that it is indeed different lifestyle behaviours and not countries heterogeneity that create this variation in the trajectories. In addition, from a methodological point of view, the fact that we considered study effect, within-country household wealth quintile, and education level in the creation of our models also enforces the belief that any heterogeneity in the study level has already been accounted for in the models specification.

These consistent findings suggest the potential for physical activity to increase baseline health and functioning and minimise decline rates in older age. Even a small amount of physical activity (such as getting involved in moderate physical activity once per week) may reduce likelihood of experiencing severe deterioration of health and functioning in older age. Among other potential pathways through which physical activity may have a beneficial effect on older adults' health, previous evidence has suggested that active older adults, compared to inactive ones, present lower cardiometabolic risk [26, 27] and age-related inflammation [28], both of which are related to many chronic diseases.

Whilst most of the population exhibited a stable trajectory at a high level of health, we found two subgroups with alternative trajectories: one with a stable lower level of health; and a third subgroup starting at a similar point as the majority of the population but whose health severely deteriorated over time. These results were similar to those reported in previous studies in terms of the number and shape of the different trajectories [9, 29, 30], although in some of these studies the declining trajectories comprised a higher percentage of the population. Our findings were replicated in the sensitivity analyses, supporting the existence of heterogeneity in ageing trajectories and the importance of accounting for this heterogeneity when studying healthy ageing $[3,4]$.

\section{Public health implications}

Empirical evidence from this study highlight the positive impact of physical activity on supporting healthy ageing, in particular the opportunity to attenuate health and functioning declines in older age. Increasing any levels of physical activity or breaking sedentary behaviour can be beneficial to health and functioning in older adults [31,32]. Indeed, physical activity promotion has been a key focus of public health policies particularly in high income countries [33]. Several community-based and web-based interventions have been developed to promote physical activity and prevent loss of functional ability in older people [25, 34-40]. However, effectiveness of physical activity interventions might be uncertain given short follow-up periods and highly selected populations in most trials [41]. Future research may assess long-term effects of physical activity interventions and identify barriers to maintain activity levels and reduce sedentary lifestyle in older age. Utilising existing cohort data with longitudinal study designs can be a possible approach to investigate trajectories of physical activity across the life course and provide evidence to identify key factors that support physical activity habits in older adults and inform populationlevel interventions [42].

Public health policies on physical activity promotion can play a key role in reducing burdens of disability as well as healthcare costs in ageing populations. Although policies that promote individual-based interventions may be helpful to develop plans and approaches to increase physical activity at the individual level, it is also important to provide a supportive environment for active ageing [43] and address environmental and social factors related to physical activity in older age [44].

\section{Conclusions}

In this study, we investigated the association between physical activity and different types of healthy ageing trajectories using a large harmonised dataset of eight cohort studies. Abstinence from any form of physical activity was associated with poor healthy ageing trajectories in terms of low baseline scores and fast decline rates. Promoting an active lifestyle appears to play an important role in maintaining health and functioning in older age. 


\section{Supplementary information}

Supplementary information accompanies this paper at https://doi.org/10. 1186/s12966-020-00995-8.

Additional file 1. Additional details on methods and results.

\section{Abbreviations}

ALSA: Australian longitudinal study of ageing; ATHLOS: Ageing trajectories of health: longitudinal opportunities and synergies; BIC: Bayesian information criterion; ELSA: English longitudinal study of ageing; ENRICA: Study on cardiovascular health, nutrition and frailty in older adults in Spain; GMM: Growth mixture modelling; HRS: Health and retirement study; JSTAR: Japanese Study of ageing and retirement; KLOSA: Korean longitudinal study of ageing; LMR-LRT: Lo-Mendel-Rubin likelihood ratio test; MAR: Missing at random; MHAS: Mexican health and aging study; MLR: Maximum likelihood with errors robust to non-normality and nonindependence of observations; SABIC: Sample-size adjusted bayesian information criterion; SHARE: Survey of health ageing and retirement in Europe; WHO: World health organization

\section{Acknowledgements}

The authors thank the ATHLOS Consortium for their support and acknowledge the funding and work of people that made possible the cohort studies whose data has been analysed in this paper.

\section{Authors' contributions}

D.M.A. and C.D. conceived the work, performed the analyses, and wrote the first draft. Y-T.W. supervised the analyses. Y-T.W., A.K., J.M.H., S.T., D.B.P., M.P., and A.M.P. provided critical feedback and contributed to the interpretation of the findings and writing of the final manuscript. All authors read and approved the final manuscript.

\section{Funding}

This work was supported by the five-year Ageing Trajectories of Health: Longitudinal Opportunities and Synergies (ATHLOS) project. The ATHLOS project has received funding from the European Union's Horizon 2020 research and innovation programme under grant agreement No 635316. The ATHLOS project researchers are grateful for data contribution and funding in the following studies:

The Australian Longitudinal Study of Ageing (ALSA): The ALSA study was supported by grants from the South Australian Health Commission, the Australian Rotary Health Research Fund, the US National Institute on Aging (Grant No. AG 08523-02), the Office for the Ageing (SA), Elderly Citizens Homes (SA), the National Health and Medical Research Council (NH\&MRC 22922), the Premiers Science Research Fund (SA) and the Australian Research Council (DP0879152; DP130100428). The authors gratefully acknowledge the work of the project team at the Flinders Centre for Ageing Studies, Flinders University who provided data for this paper.

The Seniors-ENRICA: The Seniors-ENRICA cohort was funded by an unconditional grant from Sanofi-Aventis, the Ministry of Health of Spain, FIS grant 12/ 1166 (State Secretary for R + D and FEDER-FSE) and the Centro de Investigación Biomédica en Red de Epidemiología y Salud Pública (CIBERESP), Instituto de Salud Carlos III. The authors gratefully acknowledge the work of the project team at the Universidad Autónoma de Madrid who provided data for this paper.

The English Longitudinal Study of Ageing (ELSA): ELSA is supported by the U.S. National Institute of Aging, the National Centre for Social Research, the University College London (UCL) and the Institute for Fiscal Studies. The authors gratefully acknowledge the UK Data Service and UCL who provided data for this paper.

Health and Retirement Study (HRS): The HRS study is supported by the National Institute on Aging (grant number NIA U01AG009740) and the Social Security Administration, and is conducted by the University of Michigan. The authors gratefully acknowledge the University of Michigan who provided data for this paper

The Japanese Study of Aging and Retirement (JSTAR): The JSTAR is conducted by the Research Institute of Economy, Trade and Industry (RIETI), the Hitotsubashi University, and the University of Tokyo. The authors gratefully acknowledge the RIETI who provided data for this paper.
The Korean Longitudinal Study of Ageing (KLOSA): The KLOSA study is funded by the Korea Employment Information Service (KEIS) and was supported by the Korea Labor Institute's KLOSA Team. The authors gratefully acknowledge the KEIS who provided data for this paper.

The Mexican Health and Aging Study (MHAS): The MHAS study is partly sponsored by the National Institutes of Health/National Institute on Aging (grant number NIH R01AG018016) and the INEGI in Mexico. The authors gratefully acknowledge the MHAS team who provided data for this paper retrieved from www.MHASweb.org

The Survey of Health, Ageing and Retirement in Europe (SHARE): The SHARE study is funded by the European Commission through FP5 (QLK6-CT-200100360), FP6 (SHARE-I3: RII-CT-2006-062193, COMPARE: CIT5-CT-2005-028857, SHARELIFE: CIT4-CT-2006-028812) and FP7 (SHARE-PREP: №211909, SHARELEAP: N²27822, SHARE M4: N²61982). Additional funding from the German Ministry of Education and Research, the Max Planck Society for the Advancement of Science, the U.S. National Institute on Aging (U01_AG09740-13S2, P01_AG005842, P01_AG08291, P30_AG12815, R21_AG025169, Y1-AG-455301, IAG_BSR06-11, OGHA_04-064, HHSN271201300071C) and from various national funding sources is gratefully acknowledged (see www.share-project. org).

A.M.P. was supported by the MRC (grant number: MR/K021907/1). The funders had no role in study design, data collection and analysis, decision to publish, or preparation of the manuscript.

\section{Availability of data and materials}

The datasets used and/or analysed during the current study are available from the corresponding author on reasonable request.

\section{Ethics approval and consent to participate}

The present study comprises a set of secondary data analyses using deidentified data from primary research studies, most of which are publicly available. All primary research studies received ethics approval from their corresponding local institutional review boards. Participants from all studies provided their informed consent. The ATHLOS project was approved by the Ethics Review Committee of Parc Sanitari Sant Joan de Déu, Barcelona, Spain (635316-2).

\section{Consent for publication}

Not applicable.

\section{Competing interests}

The authors declare that they have no competing interests.

\section{Author details}

${ }^{1}$ Department of Health Service and Population Research, King's College London, Institute of Psychiatry, Psychology and Neuroscience, David Goldberg Centre, De Crespigny Park, London SE5 8AF, UK. ²Department of Infectious Disease Epidemiology, London School of Hygiene \& Tropical Medicine, Faculty of Epidemiology and Population Health, London, UK. ${ }^{3}$ Parc Sanitari Sant Joan de Déu, Universitat de Barcelona. Fundació Sant Joan de Déu, Dr Antoni Pujades, 42, 08830, Sant Boi de Llobregat, Barcelona, Spain. ${ }^{4}$ Instituto de Salud Carlos III, Centro de Investigación Biomédica en Red de Salud Mental, CIBERSAM, Madrid, Spain. ${ }^{5}$ Department of Nutrition and Dietetics, School of Health Science and Education, Harokopio University, Athens, Greece.

Received: 5 March 2020 Accepted: 7 July 2020

Published online: 16 July 2020

\section{References}

1. Clegg A, Young J, lliffe S, Rikkert MO, Rockwood K. Frailty in elderly people. Lancet. 2013;381(9868):752-62.

2. Guzman-Castillo M, Ahmadi-Abhari S, Bandosz P, Capewell S, Steptoe A, Singh-Manoux A, et al. Forecasted trends in disability and life expectancy in England and Wales up to 2025: a modelling study. Lancet Public Health. 2017;2(7):e307-e13.

3. World Health Organization. World report on ageing and health. Geneva: World Health Organization; 2015.

4. Lowsky DJ, Olshansky SJ, Bhattacharya J, Goldman DP. Heterogeneity in healthy aging. J Gerontol A Biol Sci Med Sci. 2014;69(6):640-9. 
5. Langhammer B, Bergland A, Rydwik E. The importance of physical activity exercise among older people. Biomed Res Int. 2018;2018:7856823.

6. Carlson SA, Fulton JE, Pratt M, Yang Z, Adams EK. Inadequate physical activity and health care expenditures in the United States. Prog Cardiovasc Dis. 2015;57(4):315-23.

7. Gellert P, Wienert J, Ziegelmann JP, Kuhlmey A. Profiles of physical activity biographies in relation to life and aging satisfaction in older adults: longitudinal findings. Eur Rev Aging Phys Act. 2019;16:14

8. Tampubolon G. Trajectories of the healthy ageing phenotype among middle-aged and older Britons, 2004-2013. Maturitas. 2016;88:9-15.

9. Daskalopoulou C, Koukounari A, Wu YT, Terrera GM, Caballero FF, de la Fuente J, et al. Healthy ageing trajectories and lifestyle behaviour: the Mexican health and aging study. Sci Rep. 2019;9(1):11041.

10. Zaninotto $P$, Batty GD, Allerhand $M$, Deary IJ. Cognitive function trajectories and their determinants in older people: 8 years of follow-up in the English longitudinal study of ageing. J Epidemiol Community Health. 2018;72(8):685.

11. Rogers NT, Marshall A, Roberts CH, Demakakos P, Steptoe A, Scholes S. Physical activity and trajectories of frailty among older adults: Evidence from the English Longitudinal Study of Ageing. PloS One. 2017;12(2):e0170878-e.

12. Daskalopoulou C, Stubbs B, Kralj C, Koukounari A, Prince M, Prina AM. Physical activity and healthy ageing: a systematic review and meta-analysis of longitudinal cohort studies. Ageing Res Rev. 2017;38:6-17.

13. Sanchez-Niubo A, Egea-Cortés L, Olaya B, Caballero FF, Ayuso-Mateos $J \mathrm{~L}$, Prina $\mathrm{M}$, et al. Cohort profile: the ageing trajectories of health longitudinal opportunities and synergies (ATHLOS) project. Int J Epidemiol. 2019.

14. Fortier I, Raina P, Van den Heuvel ER, Griffith LE, Craig C, Saliba M, et al. Maelstrom research guidelines for rigorous retrospective data harmonization. Int J Epidemiol. 2017;46(1):103-5.

15. Salomon JA, Mathers CD, Chatterji S, Sadana R, Ustun TB, Murray CJ. Quantifying individual levels of health: definitions, concepts and measurement issues. In: Murray C, Evans D, editors. Health systems performance assessment: debates, methods and empiricism. Geneva: World Health Organization; 2003. p. 301-18.

16. Ram N, Grimm KJ. Growth mixture modeling: a method for identifying differences in longitudinal change among unobserved groups. Int J Behav Dev. 2009;33(6):565-76.

17. Jung T, Wickrama KAS. An introduction to latent class growth analysis and growth mixture modeling. Soc Personal Psychol Compass. 2008;2(1):302-17.

18. Nylund KL, Asparouhov T, Muthén BO. Deciding on the number of classes in latent class analysis and growth mixture modeling: a Monte Carlo simulation study. Struct Equ Model Multidiscip J. 2007;14(4):535-69.

19. Muthén LK, Muthén BO. Mplus User's guide. Eight ed. Los Angeles: Muthén \& Muthén; 1998-2017.

20. Vermunt JK. Latent class modeling with covariates: two improved three-step approaches. Polit Anal. 2010;18(4):450-69.

21. Asparouhov T, Muthén B. Auxiliary variables in mixture modeling: three-step approaches using Mplus. Struct Equ Model Multidiscip J. 2014;21(3):329-41.

22. van de Schoot R, Sijbrandij M, Winter SD, Depaoli S, Vermunt JK. The GRoLTS-checklist: guidelines for reporting on latent trajectory studies. Struct Equ Model Multidiscip J. 2017;24(3):451-67.

23. Gopinath B, Russell J, Kifley A, Flood VM, Mitchell P. Adherence to dietary guidelines and successful aging over 10 years. J Gerontol A Biol Sci Med Sci. 2016;71(3):349-55,

24. Daskalopoulou C, Koukounari A, Ayuso-Mateos JL, Prince M, Prina AM. Associations of lifestyle behaviour and healthy ageing in five Latin American and the Caribbean countries-a 10/66 population-based cohort study. Nutrients. 2018;10(11):1593.

25. Macera CA, Cavanaugh A, Bellettiere J. State of the art review: physical activity and older adults. Am J Lifestyle Med. 2017;11(1):42-57.

26. Stamatakis E, Davis M, Stathi A, Hamer M. Associations between multiple indicators of objectively-measured and self-reported sedentary behaviour and cardiometabolic risk in older adults. Prev Med. 2012; 54(1):82-7.

27. Gennuso KP, Gangnon RE, Matthews CE, Thraen-Borowski KM, Colbert LH. Sedentary behavior, physical activity, and markers of health in older adults. Med Sci Sports Exerc. 2013;45(8):1493-500.

28. Woods JA, Wilund KR, Martin SA, Kistler BM. Exercise, inflammation and aging. Aging Dis. 2012;3(1):130-40.

29. de la Fuente J, Caballero FF, Sánchez-Niubó A, Panagiotakos DB, Prina AM, Arndt $\mathrm{H}$, et al. Determinants of health trajectories in England and the
United States: an approach to identify different patterns of healthy aging. J Gerontol A. 2018;73(11):1512-8.

30. Browning CJ, Enticott JC, Thomas SA, Kendig HAL. Trajectories of ageing well among older Australians: a 16-year longitudinal study. Ageing Soc. 2017;38(8):1581-602.

31. de Rezende LF, Rodrigues Lopes M, Rey-Lopez JP, Matsudo VK, Luiz OC. Sedentary behavior and health outcomes: an overview of systematic reviews. PLoS One. 2014;9(8):e105620.

32. Dogra S, Stathokostas L. Sedentary behavior and physical activity are independent predictors of successful aging in middle-aged and older adults. J Aging Res. 2012;2012:190654.

33. UK government. 2019 [Available from: https:/www.gov.uk/government/ publications/physical-activity-applying-all-our-health/physical-activityapplying-all-our-health.

34. Stewart AL, Verboncoeur CJ, McLellan BY, Gillis DE, Rush S, Mills KM, et al. Physical activity outcomes of CHAMPS II: a physical activity promotion program for older adults. J Gerontol A Biol Sci Med Sci. 2001;56(8):M465-70.

35. Neidrick TJ, Fick DM, Loeb SJ. Physical activity promotion in primary care targeting the older adult. J Am Acad Nurse Pract. 2012;24(7):405-16.

36. McPhee JS, French DP, Jackson D, Nazroo J, Pendleton N, Degens H. Physical activity in older age: perspectives for healthy ageing and frailty. Biogerontology. 2016;17(3):567-80.

37. King AC. Interventions to promote physical activity by older adults. J Gerontol A Biol Sci Med Sci. 2001;56 Spec No 2:36-46.

38. Fielding RA, Guralnik JM, King AC, Pahor M, McDermott MM, Tudor-Locke C, et al. Dose of physical activity, physical functioning and disability risk in mobility-limited older adults: results from the LIFE study randomized trial. PLoS One. 2017;12(8):e0182155.

39. Davies CA, Spence JC, Vandelanotte C, Caperchione CM, Mummery WK. Meta-analysis of internet-delivered interventions to increase physical activity levels. Int J Behav Nutr Phys Act. 2012;9:52.

40. Foster C, Richards J, Thorogood M, Hillsdon M. Remote and web 2.0 interventions for promoting physical activity. Cochrane Database Syst Rev. 2013;(9). Art. No.: CD010395. https://doi.org/10.1002/14651858.CD010395. pub2.

41. Orrow G, Kinmonth AL, Sanderson S, Sutton S. Effectiveness of physical activity promotion based in primary care: systematic review and metaanalysis of randomised controlled trials. BMJ. 2012;344:e1389.

42. Lounassalo I, Salin K, Kankaanpaa A, Hirvensalo M, Palomaki S, Tolvanen A, et al. Distinct trajectories of physical activity and related factors during the life course in the general population: a systematic review. BMC Public Health. 2019;19(1):271.

43. World Health Organization. Active ageing: a policy framework. Geneva: World Health Organization; 2002

44. European Commission. Innovation for age-friendly buildings, cities and environments. A compilation of good practices 2013.

\section{Publisher's Note}

Springer Nature remains neutral with regard to jurisdictional claims in published maps and institutional affiliations.

Ready to submit your research? Choose BMC and benefit from:

- fast, convenient online submission

- thorough peer review by experienced researchers in your field

- rapid publication on acceptance

- support for research data, including large and complex data types

- gold Open Access which fosters wider collaboration and increased citations

- maximum visibility for your research: over $100 \mathrm{M}$ website views per year

At $\mathrm{BMC}$, research is always in progress.

Learn more biomedcentral.com/submissions 